\title{
Application of the Childhood Autism Rating Scale to Grasp Autistic Characteristics of the Trainees with Intellectual Disabilities in Agricultural Vocational Training
}

\author{
Masahiro Toyoda*, Yuko Yokota, Midori Kaneko \\ Graduate School of Landscape Design and Management, University of Hyogo, Kobe, Japan \\ Email: *masahiro_toyoda@awaji.ac.jp
}

How to cite this paper: Toyoda, M., Yokota, Y. and Kaneko, M. (2019) Application of the Childhood Autism Rating Scale to Grasp Autistic Characteristics of the Trainees with Intellectual Disabilities in Agricultural Vocational Training. Journal of Behavioral and Brain Science, 9, 301-312. https://doi.org/10.4236/jbbs.2019.98022

Received: July 1, 2019

Accepted: August 5, 2019

Published: August 8, 2019

Copyright $\odot 2019$ by author(s) and Scientific Research Publishing Inc. This work is licensed under the Creative Commons Attribution International License (CC BY 4.0).

http://creativecommons.org/licenses/by/4.0/

\begin{abstract}
The purpose of this study was to propose indicators for grasping autistic characteristics of people with intellectual disabilities who were going to engage in agricultural vocational training. We analyzed the data of the Childhood Autism Rating Scale (CARS) of 44 students at the high school course of a special needs education school who participated in agricultural vocational training. The data were divided into two groups according to the scores of "General Impressions", one of 15 question items of the CARS: one was the group whose score was 1 (usual), and the other was the group whose scores were 1.5 to 4 (unusual). The results of the Mann-Whitney $U$ test showed significant differences between two groups in eight items including "Relating to People" $(p<0.05)$. The Spearman's rank correlation coefficient revealed positive correlations of "Relating to People" with six other items. As a result, "Relating to People" was considered to be the most effective item to know the participant's autistic characteristics at a very early stage of training. Eventually, adding two items, "Body Use" and "Imitation" which are necessary to master agricultural tasks, to "Relating to People", we proposed three indicators.
\end{abstract}

\section{Keywords}

Agricultural Vocational Training, CARS (Childhood Autism Rating Scale), Behavioral Observation, Cooperation between Agriculture and Welfare

\section{Introduction}

In Japan, revision of legislation was made such as establishment of the special subsidiary company system for promoting employment of people with disabili- 
ties in Act on Employment Promotion etc. of Persons with Disabilities (1987) and raising of the statutory employment rate for people with disabilities to $2.2 \%$ (2018). In parallel with such revisions of the legislation, nationwide efforts to promote the employment of people with disabilities have been made in the field of agriculture which has problems of aging and lack of farmers [1]. Basic Policy on Economic and Fiscal Management and Reform 2015 was built in support for employment promotion and job retention for active participation of people with disabilities, including in the agricultural field [2]. Thus, practical actions of cooperation between agriculture and welfare started. Toyoda et al. [3] pointed out the importance of matching between the ability of people with intellectual disabilities and agricultural tasks provided to them. They also proposed an easy-to-use assessment method for analyzing and determining the difficulty of agricultural tasks [3].

Among people with intellectual disabilities, some have autism and others have some sort of autistic characteristics that may not be diagnosed as autism. Students with autism or autistic characteristics in schools for intellectually handicapped children (special needs schools) are estimated to be $25 \%$ of the students in the elementary course, the junior high school course and the high school course of special needs education schools [4]. In general, autistic characteristics appear in relationship to others, verbal communication, nonverbal communication, attitude and remarks to others, state of mind, behavioral features (e.g. how to use the body and how to use objects/tools), sensory response and so on.

In agricultural vocational training for people with intellectual disabilities, support with understanding of the trainees' autistic characteristics individually contributes not only to stress reduction, risk avoidance on the job and improvement of work efficiency, but also leads to the establishment of mutual trust relationships and continuous employment. In agricultural tasks, all autistic characteristics do not necessarily become obstacles to do the tasks. For example, there are some scenes which do not necessarily require many conversations at the stage of learning tasks or on the job. In such cases, it is considered that some sort of autistic tendencies in verbal communication do not have a major impact on work. If such facts are widely understood by the trainers of agricultural vocational training for people with intellectual disabilities or employers, the possibility for people with autism or autistic characteristics of working in the agricultural field will increase.

In this study, we assessed autistic characteristics of the students at the high school course of a special needs education school who voluntarily participated in agricultural vocational training using the Childhood Autism Rating Scale (CARS), Japanese version [5] and analyzed their CARS scores. The CARS assesses 15 items concerning a person's autistic tendencies by observing the person's behavior in his/her daily life.

The objective of this study was, based on the CARS, to propose essential indicators for grasping autistic characteristics of people with intellectual disabilities 
and simple assessment methods on the job to employers in the agricultural field and trainers of agricultural vocational training.

\section{Materials and Methods}

This study procedure was reviewed and approved by the Ethics Committee in the Graduate School of Landscape Design and Management, University of Hyogo. After oral and written explanation of the study objectives and procedures, written informed consent was obtained from every student's parents.

\subsection{Participants}

Participants were 44 students at the high school course of a special needs education school who voluntarily participated in agricultural vocational training [37 male students, (mean \pm SD) $17.11 \pm 0.91$ years; 7 female students, (mean \pm SD) $17.29 \pm 0.76$ years]. All the students who applied for the training with their parents' agreement were accepted as the participants without setting exclusion conditions. The agricultural vocational training was conducted for about three hours in the morning and for about two hours in the afternoon per day in local farms and agricultural companies. The duration was for one to three days. Among the participants, students who could perform their agricultural tasks with one-to-one instructions and those who could do the tasks almost independently, solely or in a small group of two to four members were included.

\subsection{Protocol}

During the four years of the survey (December 2011 to November 2015), every time a new student participated in agricultural vocational training, the autistic characteristics of each student was assessed using the CARS. We analyzed the CARS scores item by item and examined the inter-item correlations. Statistical analyses were performed using Excel 2010 (Microsoft, USA) with the add-in software SSRI, Version 1.02, 2012, with $p<0.05$ considered statistically significant.

\subsubsection{Assessment of the Participants' Autistic Characteristics}

Homeroom teachers or teachers in charge of career guidance at the special needs education school assessed each student's autistic characteristics using the CARS based on direct behavioral observation of the student's daily life.

The CARS comprises of 15 question items: Relating to people, Imitation, Emotional response, Body use, Object use, Adaptation to change, Visual response, Listening response, Taste, smell, and touch response and use, Fear and nervousness, Verbal communication, Nonverbal communication, Activity level, Level and consistency of intellectual functioning, and General impressions. Fifteen items are rated from 1 to 4 points each in increments of 0.5 point. One point is considered to be within the normal range compared with the behavior of standard children and two points indicate the behavior is mildly abnormal, three 
points moderately abnormal, and four points severely abnormal. In addition, 1.5 points, 2.5 points, 3.5 points indicate that the behavior is rated intermediate between normal and mildly abnormal, mildly abnormal and moderately abnormal, and moderately abnormal and severely abnormal respectively. The total minimum score is 15 points and the maximum score is 60 points. In the CARS, the total score 15 - 29.5 points are classified as "not autism", $30-36.5$ points, as "mild/moderate autism", and 37 - 60.0 points, as "severe autism" [5].

Concerning reliability of the CARS, a coefficient alpha was 0.94 and an average inter-rater correlation coefficient was 0.71 . That indicates a high degree of internal consistency and a high degree of inter-rater reliability respectively [6]. In addition, as the result of retests conducted after two years and three years, test-retest reliability coefficient was $0.88(p<0.01)$. Concerning criterion related validity, the correlation coefficient between the CARS scores and clinicians' rating was $r=0.84(p<0.001)$, and that between the CARS scores and clinical assessments by a child psychiatrist and a child psychologist was $r=0.80(p<$ 0.001), giving support for the CARS [5]. Although the CARS is applicable to children including preschool children, Mesibov et al. (1989) suggested that the CARS can also be a useful screening instrument for assessing adolescents and adults with autism [7].

In our study, the results of the total CARS scores showed 42 participants ( 35 males and 7 females) were classified as "without autism" and 2 participants (2 males and 0 females), as "with mild/moderate autism". The total CARS score of the 2 male participants was 34.5 and 35 respectively. The mean score of the whole participants was $19.9 \pm 4.8$. The minimum score was 15 and the maximum score was 35 .

If a rater feels something unusual about the target student's behavior compared with the standard children's behavioral characteristics of the same age, to know what behavioral characteristics induced such a feeling of strangeness provides a clue to clarify individual autistic characteristics.

\subsubsection{Analysis of the CARS Scores}

1) Extract of items that were characteristic of the students with autistic tendencies

First, we focused on one of the question items General impressions. The item, General impressions comprehensively assesses the degree of autism of the target student as defined in the other 14 items based on the rater's subjective impression. We divided 44 participants into two groups according to the scores of General impressions: Group I was a group of 26 students $(23$ males and $3 \mathrm{fe}-$ males) whose General impressions score was 1 point which indicates that the behavior was considered to be within the normal range compared with standard children of the same age. The mean total score of Group I was $17.0 \pm 1.4$. The minimum total score was 15 and the maximum total score was 20 .

Group II was a group of 18 students (14 males and 4 females) whose General impression scores were 1.5 points or over which indicates that the behavior was 
considered to be out of the normal range compared with standard children of the same age. The breakdown is as follows: 1.5 points indicating very slightly unusual behavior [ 8 students ( 6 males and 2 females)], 2 points indicating mildly unusual behavior [ 6 students ( 5 males and 1 female)], 2.5 points indicating intermediate between mildly unusual and moderately unusual behavior [ 3 students ( 2 males and 1 female)] and 3 points indicating moderately unusual behavior [ 1 student ( 1 male and 0 females)]. There were no students whose score was 3.5 points or over. The mean total score of Group II was $24.1 \pm 4.8$. The minimum total score was 18 and the maximum total score was 35 . Then, with regard to the 14 question items excluding General impressions, the CARS scores of the students in groups I and II were compared using the two-sided Mann-Whitney's U test $(p<0.05)$.

2) Inter-item correlations

If there is a high degree of correlation between specific question itemsofthe CARS, to check one of the correlated items or a counterpart enables to predict presence or absence of autistic characteristics in other items to some extent. We calculated the Spearman's rank correlation coefficients among question items. (Two-sided; $p<0.05$ ).

\section{Results}

\subsection{Extract of Items That Were Characteristic of the Students with Autistic Tendencies}

As Table 1 shows, Mann-Whitney's U test demonstrated significant differences

Table 1. Comparison of the CARS scores between Group I whose rating of General Impressions was within the normal range ("usual") and Group II whose rating of General Impressions deviated from the normal range ("unusual”).

\begin{tabular}{|c|c|c|c|c|c|c|}
\hline & \multirow{2}{*}{ Question Items } & Group I $(\mathrm{n}=26)$ & Group II $(n=18)$ & \multirow{2}{*}{$\mathrm{Z}$} & \multirow{2}{*}{$p^{\mathrm{a}}$} & \\
\hline & & \multicolumn{2}{|c|}{ Mean Rank } & & & \\
\hline 1 & Relating to People & 15.67 & 33.35 & 4.72 & 0.000 & ** \\
\hline 2 & Imitation & 20.65 & 25.44 & 1.50 & 0.133 & \\
\hline 3 & Emotional Response & 15.57 & 33.50 & 4.86 & 0.000 & ** \\
\hline 4 & Body Use & 20.65 & 25.44 & 1.46 & 0.144 & \\
\hline 5 & Object Use & 19.98 & 26.50 & 2.44 & 0.015 & * \\
\hline 6 & Adaptation to Change & 15.59 & 33.47 & 5.01 & 0.000 & ** \\
\hline 7 & Visual Response & 21.09 & 24.74 & 1.54 & 0.124 & \\
\hline 8 & Listening Response & 21.09 & 24.74 & 1.54 & 0.124 & \\
\hline 9 & Taste, Smell, and Touch Response and Use & 20.91 & 25.03 & 1.54 & 0.123 & \\
\hline 10 & Fear and Nervousness & 17.17 & 30.97 & 4.05 & 0.000 & ** \\
\hline 11 & Verbal Communication & 17.30 & 30.76 & 3.60 & 0.000 & ** \\
\hline 12 & Nonverbal Communication & 19.69 & 26.97 & 2.36 & 0.018 & * \\
\hline 13 & Activity Level & 17.52 & 30.41 & 3.74 & 0.000 & $* *$ \\
\hline 14 & Level and Consistency of Intellectual Functioning & 20.35 & 25.91 & 1.50 & 0.135 & \\
\hline
\end{tabular}

a. ${ }^{*}{ }^{* *}$ represent $p<0.05, p<0.01$ respectively. 
$(p<0.05)$ between the CARS scores of Group I and Group II in eight items $(R e-$ lating to People, Emotional Response, Object Use, Adaptation to Change, Fear and Nervousness, Verbal Communication, Nonverbal Communication, Activity Level). That is, it was shown that something unusual felt by the rater compared with the standard children of the same age is specifically reflected in the above 8 items. We excluded General Expressions here because it was a classification criterion for grouping.

\subsection{Inter-Item Correlations}

We calculated Spearman's rank correlation coefficients from the CARS scores of each student item by item in order to examine inter-item correlations and extracted items showing positive correlation with other items $(r>0.4, p<0.05)$.

As Table 2 shows, Adaptation to Change was correlated with other seven items, and Relating to People, and Activity Level had correlation with other six items of the 14 items excluding General Expressions. In addition, positive correlations were found between Object Use and Body Use $(r=0.607, p=0.000)$, and Imitation and Nonverbal Communication $(r=0.492, p=0.001)$. The strongest positive correlation was detected between Relating to People and Emotional Response ( $r=0.756, p=0.000)$. Listening Response and Level and Consistency of Intellectual Functioning had no correlation with other items.

\section{Discussion}

\subsection{Selection of Essential Items to Grasp the Autistic Characteristics of the Trainees with Intellectual Disabilities in Agricultural Vocational Training}

We will consider essential items to grasp the autistic characteristics of the trainees with intellectual disabilities in agricultural vocational training from the two perspectives: One is that the items are easily assessed in the ordinary settings of agricultural vocational training and effectively detect specific autistic characteristics of the trainee, and the other is that the items contribute to know the trainee's aptitude for agricultural tasks.

\subsubsection{Required Observation Items at the First Meeting}

In agricultural vocational training, trainees usually visit the site for training (i.e. farms or companies where they are going to take agricultural vocational training) in advance or on the day. There, after exchanging greetings or simple conversation, the trainees move to the agricultural field or work area to observe the work procedure, or start a task after receiving explanation about it. Therefore, it is convenient to be able to grasp roughly the trainee's autistic characteristics in the scenes of greetings, conversations, moving to another location, and work.

Among the 8 items showing significant differences between the scores of Group I and Group II as a result of the Mann-Whitney's U test $(p<0.05)$, assessable items from the scenes of greetings and conversation at the first meeting are 4 items (Relating to People, Verbal Communication, Nonverbal Communication 
Table 2. Inter-item correlations among 14 items ${ }^{a}$ of the CARS by Spearman's rank correlation coefficients.

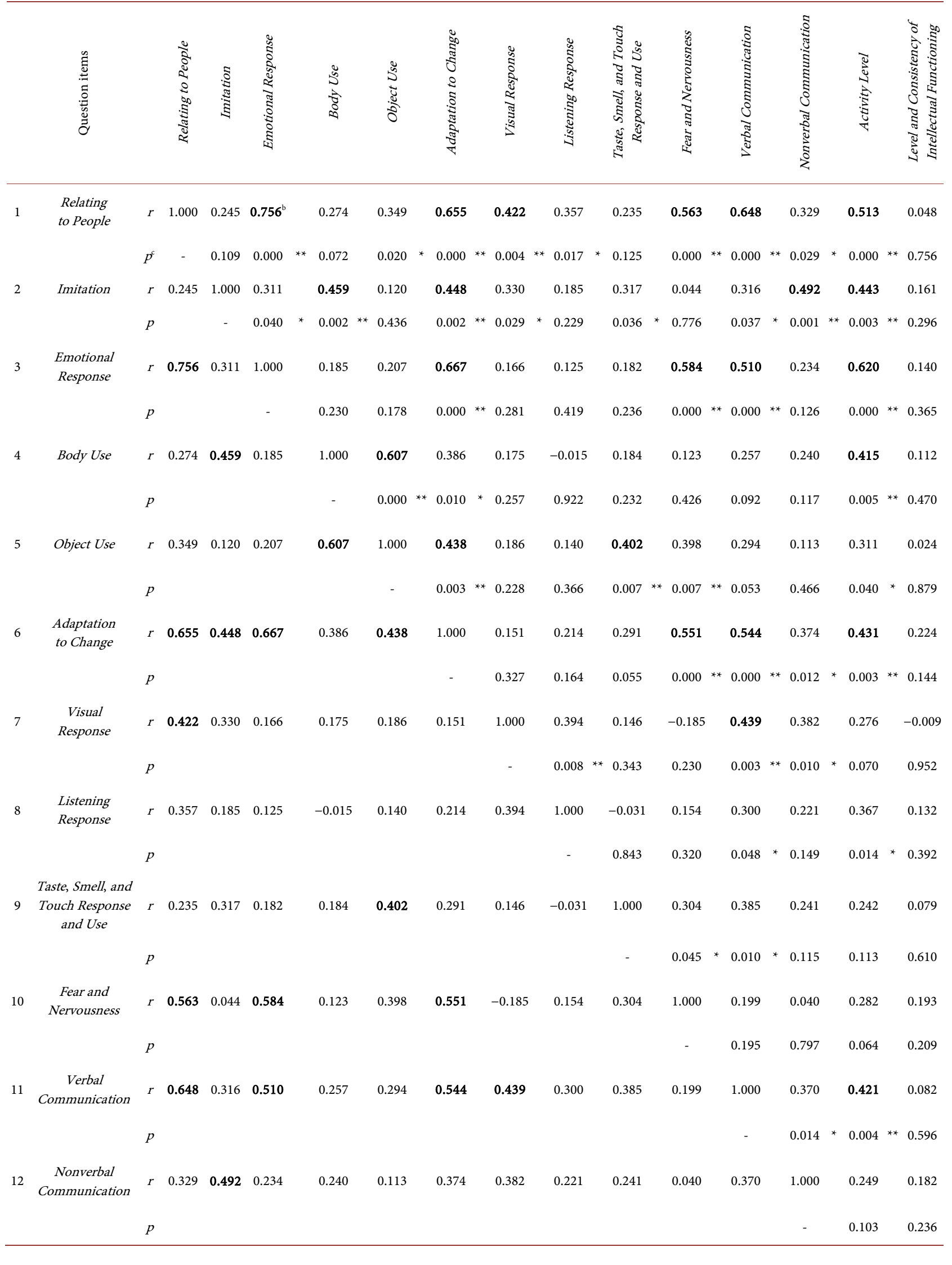




\begin{tabular}{|c|c|c|c|c|c|c|c|c|c|c|c|c|c|c|c|c|}
\hline \multicolumn{17}{|c|}{ Continued } \\
\hline 13 & Activity Level & $r$ & 0.513 & 0.443 & 0.620 & 0.415 & 0.311 & 0.431 & 0.276 & 0.367 & 0.242 & 0.282 & 0.421 & 0.249 & 1.000 & 0.222 \\
\hline \multirow{4}{*}{14} & \multirow{3}{*}{$\begin{array}{c}\text { Level and } \\
\text { Consistency } \\
\text { of Intellectual } \\
\text { Functioning }\end{array}$} & $p$ & & & & & & & & & & & & & - & 0.148 \\
\hline & & $r$ & 0.048 & 0.161 & 0.140 & 0.112 & 0.024 & 0.224 & -0.009 & 0.132 & 0.079 & 0.193 & 0.082 & 0.182 & 0.222 & 1.000 \\
\hline & & $p$ & & & & & & & & & & & & & & - \\
\hline & $\begin{array}{l}\text { The total number } \\
\text { of items where } \\
\text { a positive } \\
\text { correlation was } \\
\text { showed }(r>0.4)\end{array}$ & & 6 & 4 & 5 & 3 & 3 & 7 & 2 & 0 & 1 & 3 & 5 & 1 & 6 & 0 \\
\hline
\end{tabular}

a. The item, General Impressions was excluded in the analysis. b. Bold-type numerical values indicate the items positively correlated with each other $(r>0.4$; $p<0.05)$. c. ${ }^{*},{ }^{*}$ represent $p<0.05, p<0.01$ respectively.

and Activity Level). The remaining 4 items (Emotional response, Object Use, Adaptation to Change and Fear and Nervousness) are difficult to observe in such situations that do not involve much movement like greetings and conversation.

Among the items indicating many positive correlations with other items (Adaptation to Change, Relating to People and Activity Level), the only item that can be assessed from greetings and conversations is Relating to People. Therefore, we selected Relating to People to required observation items at the first meeting in agricultural vocational training. Concerning Relating to People, Mesibov et al. (1989) remarked that "relationships with others did not show any improvement over time on the CARS" [7].

\subsubsection{Observation Items to Grasp the Trainee's Aptitude for Agricultural Jobs}

In acquiring the skills of agricultural jobs, regardless of presence or absence of intellectual disabilities, people imitate the instructor's motions using the body and/or tools (or objects) after receiving verbal explanation and watching demonstration of the task. In their agricultural job analysis sheet for people with intellectual disabilities, Toyoda et al. (2016) mentioned that the items related to Body Use such as "necessary motions", "working posture", "use of both hands", "dexterity" and those related to Object Use such as "tools, machines and materials" are particularly important for people with intellectual disabilities to work on agricultural jobs [3]. By reference to the sheet, we picked up Body Use, Object Use and Imitation which is a necessary ability to perform a task safely and correctly as required observation items to know the trainee's aptitude for agricultural jobs. Furthermore, as Table 2 shows, a positive correlation was found between Body Use and Object Use $(r=0.607)$. If we narrow down the observation items to one, Body Use is considered to be more versatile because the item can be observed even in the scene where the trainee does not use tools. Eventually, we determined Body Use and Imitation as essential observation items to know the trainee's aptitude for agricultural jobs. 


\subsubsection{Essential Observation Items Required for Farmers (or Instructors in Charge) Who Accept Agricultural Trainees}

As the results of 4.1.2. and 4.1.3, we propose Relating to People, Body Use and Imitation as the essential observation items for farmers (or instructors) who accept agricultural trainees to grasp the trainee's autistic characteristics and aptitude for agricultural tasks (Figure 1). These three items are defined as follows [7]:

- Relating to People: This is a rating of how the child behaves in a variety of situations involving interaction with other people.

- Body Use: This scale represents a rating of both coordination and appropriateness of body movements. It includes such deviations as posturing, spinning, tapping, and rocking, toe-walking, and self-directed aggression.

- Imitation: This rating is based on how the child imitates both verbal and nonverbal acts. Behavior to be imitated should clearly be within the child's abilities. This scale is intended to be an assessment of ability to imitate, not ability to perform specific tasks or behaviors.

\subsection{Relationship between Relating to People and Other Question Items}

We validated the practicality of the item, Relating to People, as an indicator for grasping the participants' autistic characteristics. First of all, we divided the participants into two groups according to the scores of Relating to People. One was the group of 20 participants whose score was 1 and the rater felt usual about the persons' behavior (Group A: "usual”, 18 males and 2 females). The mean total score of Group A was $16.7 \pm 1.2$. The minimum total score was 15 and the

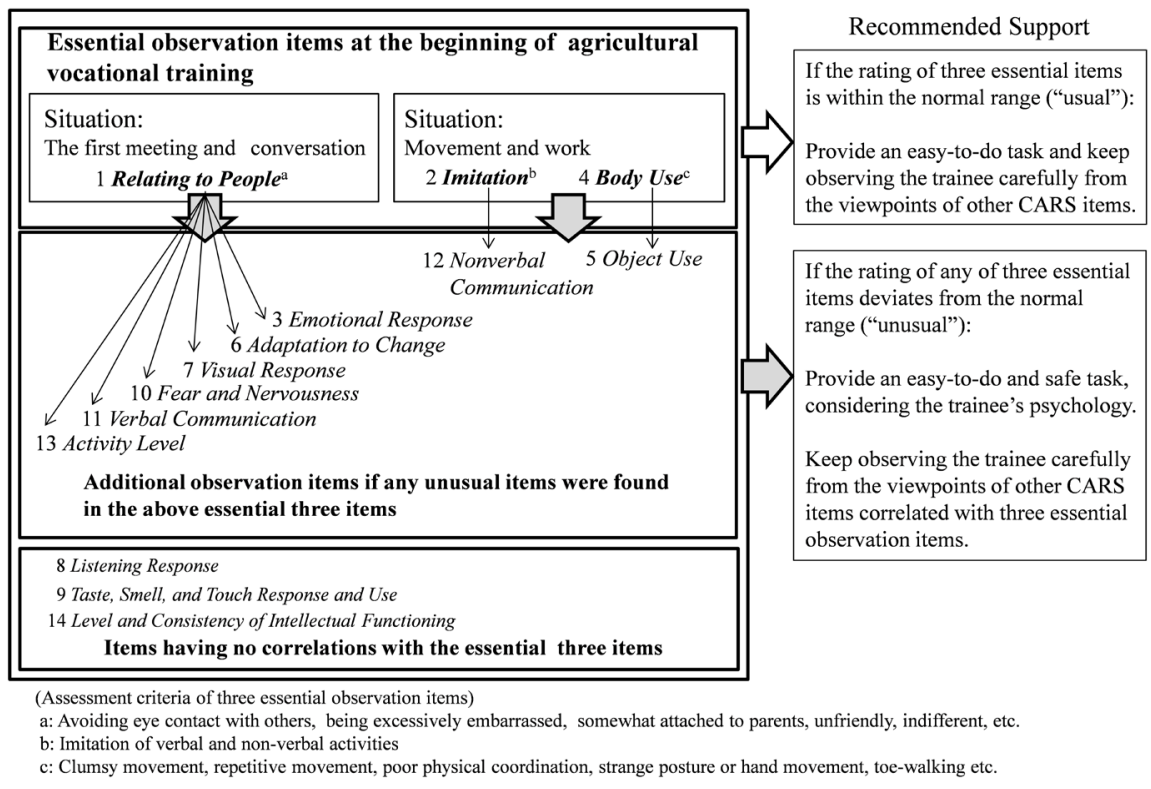

Figure 1. Relationship between scenes for grasping trainees' autistic characteristics in agricultural vocational training and 14 observation items of the Childhood Autism Rating Scale (CARS) (excluding General Impressions). 
maximum total score was 19 . The other was that of 24 participants whose scores were 1.5 or over and the rater felt something unusual about the persons' behavior (Group B: "unusual", 19 males and 5 females). The mean total score of Group B was $22.6 \pm 5.0$. The minimum score was 16.5 and the maximum score was 35. Next, we compared the percentage of the participants whose behavior was felt unusual by the rater concerning items other than Relating to People between two groups (Table 3). We conducted the two-sided hypothesis testing for the difference in the population proportions $(p<0.05)$.

The results showed that the proportion of the participants who were rated as "unusual" was significantly higher in Group B ("unusual") than Group A ("usual") in 7 items (Emotional Response, Object Use, Adaptation to Change, Fear and Nervousness, Verbal Communication, Nonverbal Communication and Activity Level) $(p<0.05)$. Comparing these seven items with the six items in which Spearman's rank correlation coefficients showed positive correlation with the score of Relating to People (except for General Impressions), 5 items were included in common: Emotional Response, Adaptation to Change, Fear and Nervousness, Verbal Communication and Activity Level. These results indicated that the observation of Relating to People has the potentiality to afford a clue to find other 5 autistic characteristics.

\section{Conclusions}

In this study, we proposed 3 major indicators (Relating to People, Body Use and Imitation) to be observed for grasping the trainees' autistic characteristics when providing the agricultural vocational training of people with intellectual disabilities. This assessment makes it easy for farmers or instructors to roughly grasp

Table 3. Itemized comparison of the proportion of the participants who were rated as "unusual" between the two groups classified by the rating of Relating to People as a classification criterion.

\begin{tabular}{|c|c|c|c|c|c|c|c|c|c|c|c|c|c|c|}
\hline 苛 & & $\begin{array}{l}\mathbb{\Xi} \\
\mathbb{\Xi} \\
\mathbb{\Xi} \\
\text { İ }\end{array}$ & 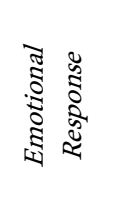 & $\begin{array}{l}0 \\
5 \\
5 \\
0 \\
0 \\
0\end{array}$ & 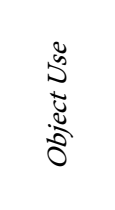 & 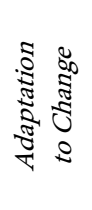 & 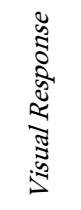 & 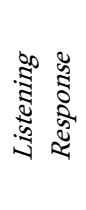 & 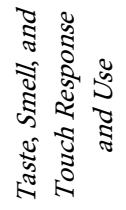 & 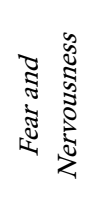 & 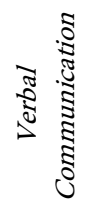 & 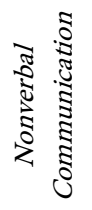 & 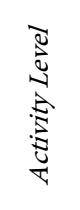 & 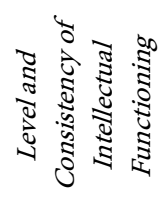 \\
\hline $\begin{array}{l}\text { Group A } \\
\text { (“usual”) }\end{array}$ & $\mathrm{n}$ & 4 & 3 & 4 & 1 & 3 & 1 & 1 & 0 & 2 & 9 & 3 & 4 & 13 \\
\hline $\mathrm{n}=20$ & $\%$ & 20.0 & 15.0 & 20.0 & 5.0 & 15.0 & 5.0 & 5.0 & 0.0 & 10.0 & 45.0 & 15.0 & 20.0 & 65.0 \\
\hline $\begin{array}{c}\text { Group B } \\
\text { (“unusual”) }\end{array}$ & $\mathrm{n}$ & 9 & 19 & 10 & 17 & 16 & 5 & 5 & 4 & 14 & 19 & 15 & 13 & 20 \\
\hline \multirow[t]{4}{*}{$\mathrm{n}=24$} & $\%$ & 37.5 & 79.2 & 41.7 & 70.8 & 66.7 & 20.8 & 20.8 & 16.7 & 58.3 & 79.2 & 62.5 & 54.2 & 83.3 \\
\hline & Z & 1.27 & 4.24 & 1.54 & 4.42 & 3.45 & 1.52 & 1.52 & 1.91 & 3.32 & 2.35 & 3.19 & 2.32 & 1.40 \\
\hline & $p^{\mathrm{b}}$ & 0.205 & $<0.0001$ & 0.124 & $<0.0001$ & 0.001 & 0.128 & 0.128 & 0.056 & 0.001 & 0.019 & 0.001 & 0.021 & 0.162 \\
\hline & & & $* *$ & & $* *$ & $* *$ & & & & $* *$ & * & $* *$ & * & \\
\hline
\end{tabular}

a. Group A ("usual") is a group whose rating of Relating to People was within the normal rangeland Group B ("unusual") is a group whose rating of Relating to People deviated from the normal range. $\mathrm{b} .{ }^{*},{ }^{* *}$ represent $p<0.05, p<0.01$ respectively. 
autistic characteristics of the trainees who wish to take agricultural vocational training, especially when there is little information about the trainees with intellectual disabilities, such as at the first meeting or at the early stage of training.

We suggest that farmers or instructors should roughly grasp the autistic characteristics of each trainee by starting with observation of these 3 items, and in the course of training, gain better understanding of his/her other autistic characteristics. It is important to provide adequate agricultural tasks or support better suited to each trainee based on proper appreciation about his/her autistic characteristics.

Our findings will provide the following advantages to those who are going to provide agricultural vocational training for people with intellectual disabilities:

1) To reduce matching errors between trainees with intellectual disabilities and provided agricultural tasks.

2) To promote mutual understanding and the establishment of relationship of trust between the two.

3) To improve work safety and work efficiency.

\section{Acknowledgements}

This study is a summary of the results of research conducted from 2011 to 2015 in cooperation with the Hyogo Prefectural Government, Health and Welfare Department, Welfare Bureau for People with Disabilities, Division for the Support of People with Disabilities and Hyogo Social Welfare Corporation. We thank teachers, parents and students of a special needs education school, all the supporters of agricultural vocational training and concerned parties for cooperation.

\section{Conflicts of Interest}

The authors declare no conflicts of interest.

\section{References}

[1] Ministry of Agriculture, Forestry and Fisheries (2013) Case Studies of Medical, Welfare, Food and Agriculture Cooperation. http://www.maff.go.jp/j/keikaku/pdf/sankou_jireishu4.pdf

[2] Cabinet Decision (2015) Basic Policy on Economic and Fiscal Management and Reform 2015. http://www5.cao.go.jp/keizai-shimon/kaigi/cabinet/2015/2015_basicpolicies_ja.pdf

[3] Toyoda, M., Kaneko, M., Yokota, Y., Asai, S., Fudano, T. and Shiroyama, Y. (2016) A New Assessment Method of Agricultural Work in Job Assistance for People with Intellectual Disabilities. Journal of the Japanese Society of People-Plant Relationships, 15, 1-10. http://jsppr.jp/academic_journal/pdf/Vol15.No2_P01-10.pdf

[4] The National Institute of Special Education Research (2010) Studies on Education for Children with Autistic Disorder 5. Tokyo.

[5] Schopler, E., Reichler, R.J. and Renner, B.R. (2012) The Childhood Autism Rating Scale: CARS (Translated into Japanese). Iwasaki Academic Publisher, Tokyo.

[6] Schopler, E., Reichler, R.J., DeVellis, R.F. and Daly, K. (1980) Toward Objective 
Classification of Childhood Autism: Childhood Autism Rating Scale (CARS). Journal of Autism and Developmental Disorders, 10, 91-103.

https://doi.org/10.1007/BF02408436

[7] Mesibov, G.B., Schopler, E., Schaffer, B. and Michal, N. (1989) Use of the Childhood Autism Rating Scale with Autistic Adolescents and Adults. Journal of the American Academy of Child \& Adolescent Psychiatry, 28, 538-541.

https://doi.org/10.1097/00004583-198907000-00012 